\title{
Sistemas de innovación y patrones de interacción local en el sector rural en México
}

\section{Innovation systems and local interaction patterns in the rural sector in Mexico}

Anastacio Espejel-García ${ }^{1}$, Ariadna Barrera-Rodríguez ${ }^{2}$, Venancio Cuevas-Reyes ${ }^{3}$, Ma. Carmen Ybarra Moncada ${ }^{4}$ y José Apolonio Venegas Venegas ${ }^{5}$

Palabras clave: fuente de innovación; interacción; actores; gestión de conocimiento

\section{Recepción: 11-01-17 / Aceptación: 11-06-17}

\section{Resumen}

El objetivo fue identificar las fuentes de innovación y patrones de interacción en dos sistemas de innovación pecuarios en México con la finalidad de proponer estrategias que permitan mejorar los procesos de aprendizaje e innovación de este tipo de sistemas. A través del estudio de dos sistemas de innovación pecuarios se obtuvieron indicadores de redes sociales que permitieran la identificación de actores y los mecanismos de interacción que siguen en la adopción de innovaciones. Los resultados encontrados muestran que los patrones de interacción responden a la necesidad de innovar y determinan dos tipos de relaciones: una dirigida y la otra liderada por la oportunidad del mercado. Existen agentes que fungen como intermediarios de la innovación con la capacidad de articular al sistema de innovación, vincular a los actores y llevar la innovación hasta el usuario final. Se concluye que los intermediarios con mayores vínculos definen las fuentes, tipos de innovación y activan los patrones y mecanismos de interacción entre los actores de los sistemas de innovación.

\footnotetext{
Abstract

The objective was to identify the sources of innovation and interaction patterns in two livestock innovation systems in Mexico with the purpose of contributing information to propose strategies to improve the learning and innovation processes of this type of systems. Through the study of two livestock innovation systems, social network indicators were obtained that allowed the identification of actors and the interaction mechanisms that follow in the adoption of innovations. ${ }^{1}$ Posgrado en Ciencia y Tecnología Agroalimentaria, Universidad Autónoma Chapingo. E-mail: aespejelga@conacyt.mx

${ }^{2}$ Preparatoria Agrícola, Universidad Autónoma Chapingo

${ }^{3}$ Campo Experimental Valle de México, Instituto Nacional de Investigaciones Forestales, Agrícolas y Pecuarias.

${ }^{4}$ Departamento de Ingeniería Agroindustrial, Universidad Autónoma Chapingo.

${ }^{5}$ Facultad de Ciencias Agronómicas, Universidad Autónoma de Chiapas

(C) Universidad De La Salle Bajío (México)
} 
The patterns of interaction respond to the need to innovate and determine two types of relationships: one directed and the other led by market opportunity. There are agents who act as intermediaries of innovation with the ability to articulate the innovation system, link the actors and bring innovation to the end user. It is concluded that the intermediaries with greater links define the sources and types of innovation activate the patterns and mechanisms of interaction between the actors of the innovation systems.

Keywords: innovation source; interaction; actors; knowledge management.

\section{Introducción}

La “innovación es un proceso complejo que deriva y depende simultáneamente de dimensiones micro, meso y macro y que involucra a diversos agentes" (Spielman et al. 2008:3; Carrillo et al. 2012; 10;). Al contar con diferentes dimensiones, la innovación como proceso involucra diversos actores, instituciones y relaciones entre ellos, todos actuando como una unidad para lograr que las innovaciones (de conocimiento o tecnológicas) logren un impacto, una mejora que se represente en mayor productividad, pero sobre todo en mayores ingresos y beneficios para las empresas o para los productores.

Se reconoce a la innovación como un proceso interactivo entre individuos y organizaciones que procesan diversos tipos de conocimiento, y que se desarrollan en un ambiente político, económico y social específico. A partir de ello se hace relevante la perspectiva sistémica y se presta mayor atención a los procesos de interacción entre la investigación y la actividad económica relacionada, las prácticas que promueven la interacción y aprendizaje que la acompaña y la creación de un ambiente facilitador de la innovación y que contribuya con la generación de conocimiento social y económicamente útil, (Banco Mundial, 2008).

El análisis del Sistema de Innovación (SI) es una herramienta útil que puede ayudar a entender los procesos de innovación y difusión del conocimiento en el sector agrícola (Lamprinopoulou et al. 2014:41). De este modo, las innovaciones surgen de las interacciones complejas que se dan entre un conjunto diverso de actores públicos, privados y de la sociedad civil que se dedican a generar, intercambiar y utilizar el conocimiento dentro del denominado Sistema de Innovación Agrícola (AIS) (Hall et al. 2004:3; Spielman et al. 2008:2). El reconocimiento de 
múltiples actores en los procesos de innovación y la interacción entre ellos ha llevado a los estudiosos de este tema y a tomadores de decisión, a darle mayor importancia a la visión sistémica de la innovación (Smits and Kuhlmann 2004:16; Voss et al. 2009:280; Raven et al. 2010:63; Van Mierlo et al. 2010:325).

Las herramientas sistémicas deben proporcionar elementos para abordar los problemas que surgen del proceso de innovación y que influyen en la velocidad y procesos de adopción de innovación (Wieczorek, et al. 2012:77). El desempeño de los sistemas de innovación está asociado a la intensidad y a la eficacia de las interacciones entre los diferentes protagonistas comprometidos en la generación y difusión de nuevos conocimientos y nuevas tecnologías (OCDE, 2002). El marco de los sistemas de innovación, según Berdegué (2005:15), abre la caja negra de la innovación para analizar las funciones de los distintos agentes de innovación, los tipos y la calidad de las interacciones entre ellos y las instituciones formales y no formales que estructuran tales procesos.

El sistema de innovación debe enfocarse en la totalidad de actores involucrados en la innovación y sus interacciones (Banco Mundial, 2008:115). De acuerdo con Spielman et al. (2011:196) dado que la innovación proviene principalmente del intercambio y uso del conocimiento, la naturaleza de las interacciones entre los actores es otro aspecto importante a considerar. Este mismo autor señala entonces que, el estudio de cómo los actores estructuran sus interacciones en el intercambio de conocimientos ofrece al marco de los sistemas de innovación su perspectiva de sistemas definitivo. De esta forma, "el desempeño de los sistemas de innovación está asociado a la intensidad y a la eficacia de las interacciones entre los diferentes protagonistas comprometidos en la generación y difusión de nuevos conocimientos y nuevas tecnologías" (OCDE, 2002:27). Los actores pueden actuar de diversas formas, sin embargo, cada uno de ellos intervendrá de forma más o menos dinámica dependiendo del nivel de colaboración y de los recursos con los que cuenta, pero más aún del rol que tiene dentro del entramado institucional y objetivos que persigan (FAO y Banco Mundial, 2000:145).

Este contexto dinámico, requiere de sistemas de innovación que consideren los actores y sus interacciones para poder lograr que las innovaciones implementadas tengan impactos perdurables en el tiempo y se reflejen en mayores impactos en las familias rurales (Santamaría, 2005 citado por FAO, 2012:34; Hall et al. 2004:3). Conocer los principales actores que interactúan en una cadena agroalimentaria permite no solo su identificación, sino conocer sus características y la forma o patrones de vinculación e interacción, lo que eventualmente puede ser utilizado para 
definir estrategias y mejorar la innovación de la cadena analizada dentro de un enfoque integral. El análisis sistémico aplicado a cadenas agroalimentarias establecidas en el territorio representa una alternativa a la visión lineal de generación y difusión de conocimiento, problema vigente en el sector agropecuario en México. Es por ello que el objetivo de este trabajo de investigación fue identificar las fuentes de innovación y patrones de interacción en dos sistemas de innovación pecuarios en México, con la finalidad de contribuir con información para proponer estrategias que permitan mejorar los procesos de aprendizaje e innovación de este tipo de sistemas. Este artículo contribuye esencialmente con el análisis de dos elementos estratégicos en la construcción de los sistemas de innovación: los actores como fuentes de innovación y aprendizaje, y los tipos de interacciones que suceden entre ellos y que configuran el sistema y permiten su desarrollo. A partir de esta premisa uno de los desafíos de la innovación en relación con los sistemas agropecuarios es expandir las oportunidades y los medios a los productores pobres en recursos y que ello contribuya

a que se conviertan en actores más activos y beneficiarios en estos sistemas de innovación. Por lo tanto, la lógica de los sistemas de innovación deberá construirse para la resolución de problemas locales y situar a la innovación no como una meta sino como una respuesta a la solución de problemas de los sistemas de producción y de la sociedad.

\section{Materiales y métodos}

\section{Área de estudio}

El presente estudio se desarrolló en dos regiones con alto potencial en la producción pecuaria, una en la producción de leche de bovino en el estado de Hidalgo, y la otra en la producción de ovinos en pie en el Estado de México. El estudio del sistema de innovación de bovinos leche, se llevó a cabo en la región denominada, Valle del Mezquital, en el estado de Hidalgo, en los municipios de Francisco I. Madero, Actopan, Ixmiquilpan y San Salvador (Figura 1), que son los que registran una mayor producción de leche (SIAP, 2013), y que de acuerdo con la Comisión Estatal de la leche (CEL, 2006:23) concentran el 48\% de los productores de la región. La región del Valle del Mezquital presenta condiciones de zona semiárida, clima de semiseco a seco, temperatura mayor de $20^{\circ} \mathrm{C}$ y presencia de lluvias menor a $700 \mathrm{~mm}$ anuales. El estudio del sistema de innovación de ovinos se llevó a cabo en los municipios de Villa Victoria y Villa de Allende en el Estado de México, debido a que cuentan con tradición en la actividad ganadera y condiciones agroecológicas para desarrollar esta actividad (Figura 1).

No 19, Vol. 9 (2), 2017. ISSN 2007 - 0705, pp.: 595 - 614 


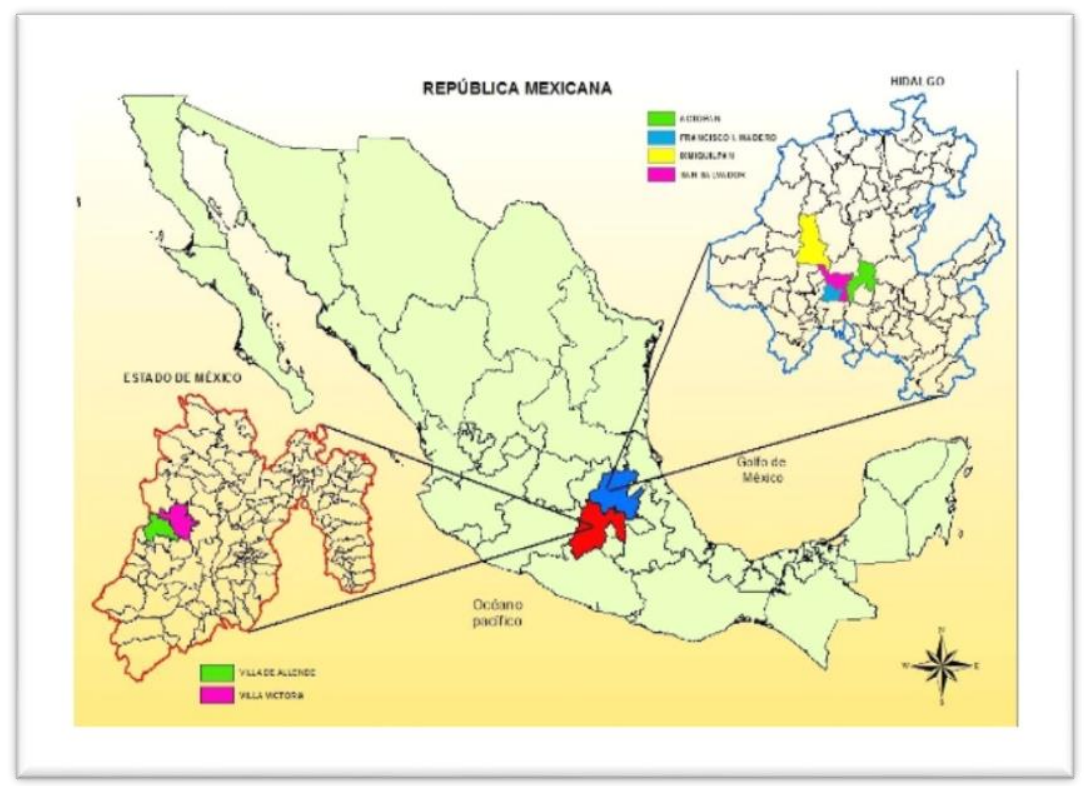

Figura 1. Localización del área de estudio.

Fuente: Elaboración propia, 2017.

\section{Diseño de muestreo}

Para el análisis de la cadena de producción de leche en el estado de Hidalgo, se empleó la base de datos y el diseño muestral de un estudio previo realizado por el autor (Espejel et al. 2016:6). Se seleccionó a 66 productores de un universo de 211 en el año 2012, mediante un muestreo simple aleatorio (Ecuación 1).

$$
n=\frac{N Z^{2} p q}{d^{2}(N-1)+Z^{2} p q}
$$

Dónde:

$\mathrm{n}=$ Número de productores a encuestar.

$\mathrm{N}=$ Número total de productores

d=Precisión (expresada en proporción): $10 \%=0.1$

$\mathrm{Z}=$ Para poblaciones mayores a 100 , la confiabilidad se puede estimar con base a los siguientes valores: $95 \%=1,96 ; 90 \%=1,64$. Para poblaciones menores a 100, recurrir a los valores de la tabla de porcentajes de la distribución t de Student.

$\mathrm{p}=$ Proporción de la población $=0,5$

$\mathrm{q}=$ Diferencial de $\mathrm{p}:(1-\mathrm{p})=0,5$ 
Para el estudio de la cadena ovinos en el Estado de México, se aplicó un muestreo por intención o dirigido, (Vivanco 2005:27) y se analizaron 60 productores (25\% del marco muestral) de un total de 242 registrados en el Programa PROGAN productivo en el año 2012, y que participaron en el programa de capacitación y asistencia técnica estatal, promovido por la Secretaría de Desarrollo Agropecuario del Estado de México dentro de la estrategia de Corredores de Desarrollo Rural (CODER) durante el ciclo 2013-2014. Los criterios de selección fueron: la disposición y compromiso a adoptar las innovaciones, contar con rebaño y superficie con potencial. La selección se realizó con base en el cumplimiento de los requisitos del Programa.

\section{Elaboración de la encuesta}

La encuesta aplicada a productores de ambas cadenas se integró con los siguientes apartados: i) datos generales del productor, edad, años de experiencia en la actividad, escolaridad; ii) dinámica de la producción, contiene información sobre la composición del rebaño para ovinos y del hato para bovinos leche; iii) dinámica de innovación, información sobre las innovaciones que adoptó el productor; se consideraron 53 innovaciones para los productores de leche y 34 innovaciones para los ovinocultores; iv) redes de innovación, considera las fuentes de aprendizaje e innovación, de donde aprendió el productor y las interacciones entre los diversos actores que participan en la adopción y difusión de innovaciones.

\section{Definición de indicadores para el análisis del Sistema de Innovación}

Con la información obtenida de la aplicación de encuestas a productores, se construyó una base de datos en Excel®, la cual posteriormente fue exportada a UCINET 6 (Borgatti et al. 2002:6) para realizar las operaciones correspondientes al cálculo de indicadores de la red y fuentes de innovación. El análisis de los patrones de interacción se realizó con base en los indicadores de centralidad, índice de cobertura, actor difusor y fuentes de innovación.

Centralidad: permite el análisis de cada actor en lo individual, mediante la estimación de las relaciones de cada uno con el resto de los actores. Se mide con los grados de entrada y salida de cada actor en términos de porcentaje (Borgatti et al. 2002:18). Los grados de entrada reflejan el número de veces que un actor es referenciado por otro. Los grados de salida expresan el número de referencias que hace un actor respecto a otro. Para fines del presente estudio sólo se empleará 
los grados de entrada de los actores. El Índice de cobertura: el indicador de cobertura se calculó a partir de identificar a los actores clave con Key Player 2; indica el grado de cobertura que tienen los actores identificados como clave dentro de la red, es decir la capacidad de un grupo de actores para llegar al resto de los actores de la red (Borgatti and Halgin, 2011:4). En el mismo sentido, El actor difusor (diffuse): se calculó empleando el algoritmo que se indica en la ecuación 1. "Se emplea en la literatura de redes la letra $\mathrm{R}$ como abreviatura de alcance. Considerando que la distancia dmj del último nodo a cualquier otro nodo es $1, \mathrm{y}$ es el total de nodos" (Rendón et al. 2007:45).

$$
R=\frac{\sum_{j} \frac{1}{d m j}}{N}
$$

Fuentes de innovación (FuIn): expresa la importancia (porcentaje) de cada actor en la red como difusor del total de innovaciones adoptadas por los productores. Se calcularon mediante la ecuación (2) (Muñoz et al. 2010:15):

$$
\text { Fuln }=\sum_{i=1}^{j}\left(\frac{\text { Fuentes de innovación del productor } i}{\text { Fuentes totales de los productores } j}\right) * 100
$$

\section{Resultados y Discusión}

Los dos sistemas de innovación analizados muestran evidencia de diferentes patrones de interacción (Banco Mundial, 2008), lo cual está determinado por las condiciones del entorno y la participación de los actores, las fuentes de innovación y el conocimiento.

\section{Fuentes de innovación}

La diversidad de actores y su apoyo para la innovación determinan el tipo de trayectoria o interacción que siguen los principales actores de los sistemas de innovación (SI) analizados. Derivado de la encuesta aplicada a productores en el apartado de redes, se encontró que el intercambio de conocimiento entre productores de ambos SI es el predominante, ya que la principal fuente de innovación para el 50\% de los productores son sus pares, los servicios de asistencia técnica tanto públicos como privados que expresan el conocimiento explicito son fuente de 
aprendizaje para el $40 \%$ de los productores. La CEL es una fuente de innovación para el 10\% de los productores; este actor ha sido el nodo más importante en el sistema de innovación y el facilitador de la innovación (Fukuda, et al. 2002:33), las instituciones de investigación tienen una cobertura baja pues sólo un $0.5 \%$ de los productores considera a estas como reservorio de conocimiento (Tabla 1).

Tabla 1. Fuentes de innovación e intercambio de conocimiento en el SI de bovinos leche.

\begin{tabular}{|c|c|c|c|}
\hline $\begin{array}{l}\text { Intermediario de la } \\
\text { innovación }\end{array}$ & Objetivo que persigue & $\begin{array}{c}\text { Área o eslabón } \\
\text { en el que } \\
\text { enfoca su } \\
\text { trabajo }\end{array}$ & $\begin{array}{c}\text { Importancia } \\
\text { relativa de la } \\
\text { fuente de } \\
\text { innovación }(\%)\end{array}$ \\
\hline Productor & $\begin{array}{c}\text { Mejorar sus condiciones de } \\
\text { vida }\end{array}$ & Producción & 49.5 \\
\hline $\begin{array}{c}\text { Servicios de } \\
\text { Asistencia Técnica } \\
\text { privados }\end{array}$ & $\begin{array}{l}\text { Transferir tecnología e } \\
\text { innovaciones y venta de } \\
\text { insumos }\end{array}$ & Producción & 18.4 \\
\hline $\begin{array}{c}\text { Servicios de } \\
\text { Asistencia Técnica } \\
\text { públicos }\end{array}$ & $\begin{array}{c}\text { Desarrollar capacidades en } \\
\text { productores }\end{array}$ & $\begin{array}{l}\text { Producción- } \\
\text { Transformación }\end{array}$ & 19.5 \\
\hline Gobierno Estatal & $\begin{array}{l}\text { Financiar proyectos } \\
\text { productivos, activos, } \\
\text { capacitación y asistencia } \\
\text { técnica a fondo perdido }\end{array}$ & $\begin{array}{l}\text { Producción- } \\
\text { Transformación }\end{array}$ & 10.2 \\
\hline $\begin{array}{l}\text { Gobierno Federal } \\
\text { (IE\&I) }\end{array}$ & $\begin{array}{c}\text { Generar paquetes } \\
\text { tecnológicos e } \\
\text { innovaciones para todas las } \\
\text { cadenas agroalimentarias }\end{array}$ & Producción & 0.5 \\
\hline $\begin{array}{l}\text { Organizaciones } \\
\text { Financieras }\end{array}$ & $\begin{array}{c}\text { Otorgar créditos a los } \\
\text { productores para fortalecer } \\
\text { las cadenas } \\
\text { agroalimentarias }\end{array}$ & $\begin{array}{l}\text { Producción- } \\
\text { Transformación }\end{array}$ & 1.9 \\
\hline
\end{tabular}

Fuente: Elaboración propia con datos obtenidos en campo, 2012.

En el Sistema de Innovación de ovinos en el Estado de México, los resultados sugieren que la innovación puede ser provocada y difundida de diferentes formas y a través de diferentes actores. Los productores resultan ser claves como reservorio de conocimiento y lo que se puede interpretar tras este comportamiento, es la confianza construida entre pares, a partir de la presencia de un agente técnico como fue la agencia de desarrollo financiada con recursos públicos (FederaciónEstado); con este actor se mejoró la difusión y con ello la adopción de innovaciones. El 96\% de la 
información se concentra en los productores y la agencia de desarrollo, y en muy baja proporción en los proveedores de insumos o compradores del producto (Tabla 2).

Tabla 2. Fuentes de innovación e intercambio de conocimiento en el SI ovinos en el Estado de

\begin{tabular}{cccc}
\hline $\begin{array}{c}\text { Intermediario de la } \\
\text { innovación }\end{array}$ & $\begin{array}{c}\text { Objetivo que } \\
\text { persigue }\end{array}$ & $\begin{array}{c}\text { Área o eslabón en } \\
\text { el que enfoca su } \\
\text { trabajo }\end{array}$ & $\begin{array}{c}\text { Importancia } \\
\text { relativa de la } \\
\text { fuente de } \\
\text { innovación (\%) }\end{array}$ \\
\hline Productor & $\begin{array}{c}\text { Incremento de sus } \\
\text { ingresos y mejora } \\
\text { de su bienestar }\end{array}$ & Producción & 56.5 \\
Proveedor de & $\begin{array}{c}\text { Venta de insumos } \\
\text { para la producción } \\
\text { ovina }\end{array}$ & Producción & 1.6 \\
insumos (Privado) & $\begin{array}{c}\text { Acopiar cordero } \\
\text { flaco, engorda y } \\
\text { venta al rastro y } \\
\text { consumidor final } \\
\text { corderos }\end{array}$ & Comercialización & \\
Servicios de AT & $\begin{array}{c}\text { Desarrollo de } \\
\text { capacidades en } \\
\text { productores, }\end{array}$ & $\begin{array}{c}\text { Transformación- } \\
\text { Comercialización }\end{array}$ & \\
de Desarrollo) & $\begin{array}{c}\text { Producción- } \\
\text { capacitación y }\end{array}$ & & \\
asistencia técnica & & \\
\hline
\end{tabular}

Fuente: Elaboración propia con datos obtenidos en campo, 2013.

Este tipo de interacciones mostradas suelen denominarse trayectorias o patrones dirigidos. Las características de éstas se resumen en tres etapas: la primera llamada de preplanificación en la cual no se ha realizado intervención alguna de investigación; fase de fundación, en donde se han identificado sectores, productos, el gobierno les apoya con intervenciones de investigación y política; fase de expansión, en la cual el gobierno interviene con proyectos y programas especiales para ligar a los actores en el sistema de innovación (FAO, 2004:67). A partir del hecho de que la innovación es un proceso social que implica la interacción de diferentes actores desde la generación, uso y difusión de innovaciones, es relevante conocer a los agentes que son fuente de innovación. Los productores son un factor clave como fuente de innovación en los sistemas analizados. Con mayor intensidad los productores de ovinos mencionaron que el $56.5 \%$ de las innovaciones las aprendieron de otro productor, en comparación con bovinos leche en donde es el 49.5\%. Este dato es un indicador relevante y sugiere que el conocimiento tácito es estratégico en 
los procesos de innovación a nivel local y con pequeños productores y representa una fortaleza en la implementación de estrategias de innovación.

El papel de las instituciones gubernamentales en sistemas de innovación en donde hay incidencia de pequeños productores es fundamental y pueden desempeñar diferentes roles. Uno de los más importantes como fuentes de aprendizaje, son los servicios de asistencia técnica. De acuerdo con Solleiro et al. (2015:1262-1263) las instituciones y organizaciones públicas son las que principalmente combinan sus recursos para la gestión de innovación, por lo tanto, tienen un papel protagónico en el sistema; sin embargo, además de los esfuerzos institucionales también incluye la voluntad del resto de los actores. En el caso de ovinos, el $40.3 \%$ de los productores mencionaron a los servicios de asistencia técnica como fuente de innovación, mientras que en bovinos leche su incidencia es más baja (19.5\%) debido a que en esta cadena tiene una participación importante el servicio de asistencia técnica privada (18.4\%) y en ovinos sólo es de 1.6\% (Figura 2). Estas diferencias radican en la especialización y demanda tecnológica de las especies y de manera importante en la vinculación que tienen con el mercado. Las instituciones de gobierno pueden fungir como fuentes importantes de tecnología y maquinaria y equipo, en la cadena ovinos se muestra la ausencia del gobierno en estos rubros, mientras que en bovinos leche aparecen el gobierno estatal (10.2\%) y el gobierno federal (0.5\%) (Figura 2).

Debido al nivel de desarrollo y una mayor demanda de tecnología en la producción de leche, se requiere del apoyo de fuentes de financiamiento privado, tal es el caso, que, aunque baja, la incidencia de instituciones financieras como fuente de innovación es del 1.9\% en bovinos leche, en tanto, en ovinos prácticamente este agente no existe. 


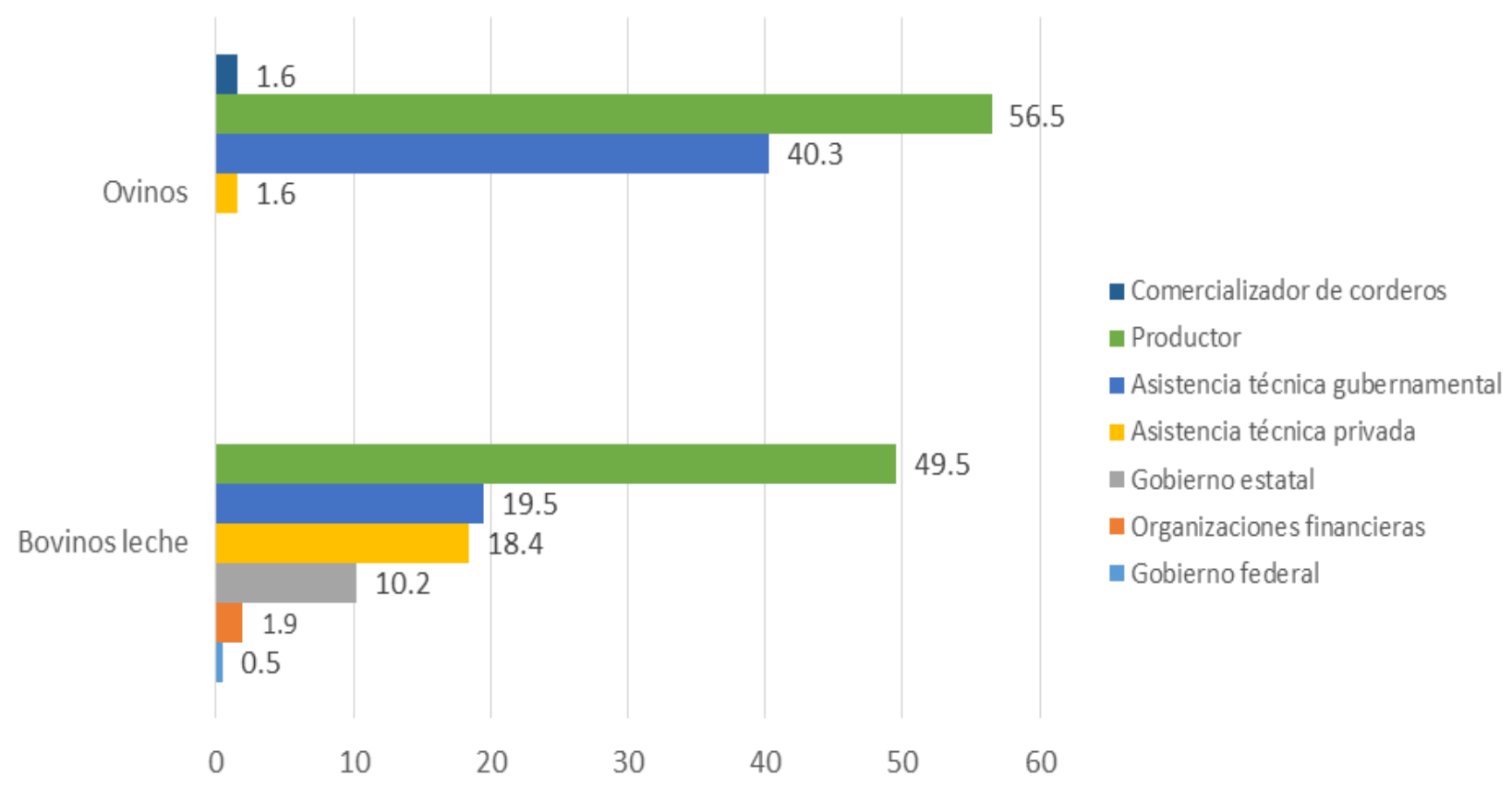

Figura 2. Frecuencia de fuentes de innovación y aprendizaje en los SI pecuarios analizados.

Fuente: Elaboración propia con base en encuestas de campo, 2012-2013.

El mercado es uno de los elementos que en los últimos años ha tenido participación importante como un motor de desarrollo para la producción primaria. En el caso de bovinos leche de manera directa no aparece como fuente de innovación, debido a que el gobierno estatal mediante la CEL actúa de manera activa como intermediario entre el industrial y el productor. Mientras que en ovinos, debido a que un porcentaje elevado de los productores comercializa el cordero al intermediario, mencionan a este actor con el 1.6\% como fuente de innovación. En un estudio realizado por Clark et al. (2006:22-25) para analizar los procesos de difusión de innovaciones y conocimiento en Bolivia en las cadenas agroalimentarias de café, melocotón y chile en Bolivia, concluyen que hay actores indispensables para el funcionamiento de las cadenas, como los comerciantes. Hay organizaciones privadas o dependencias del gobierno que fungen como transmisores de información sobre el mercado, como es el caso de FECAFEB (Federación de Exportadores de Café de Bolivia) que ha permitido contribuir al fortalecimiento del sistema de innovación en dicha cadena. De acuerdo con Spileman et al. (2011:204-205) en un estudio sobre los sistemas de innovación de pequeños productores de Etiopía, se concluye que la ausencia de comerciantes, bróker dentro del sistema, limita las acciones emprendidas por el gobierno en el tema de extensionismo. 
Los actores que participan como fuente de innovación en ambas cadenas tienen coincidencia en los grandes rubros (productores y asistencia técnica de gobierno) mientras que en los rubros con porcentajes más bajos es donde se diferencia. Esta diferenciación en las fuentes de innovación podría ser atribuida al nivel de desarrollo, dinamismo e integración al mercado, Espejel, et al. (2014:10) de las cadenas analizadas.

\section{Análisis de indicadores de redes de los Sistemas de Innovación de ovinos y bovinos leche}

El abordaje del análisis de redes ha sido considerado desde diferentes puntos de vista, como una herramienta para la identificación de actores y potencializar la transferencia de tecnología entre productores primarios, Muñoz, et al. (2004:16); como una estrategia de intervención en programas del sector rural en México con fines de extensionismo (Espejel, et al. 2014:10; Aguilar, et al. 2010:3); y, como elemento de análisis del sistema de innovación agropecuaria en México (Cuevas, et al. 2016:1298).

Con base en el análisis de redes sociales se identificó a los actores difusores del sistema en bovinos leche. Estos resultaron ser tres productores, los cuales podrían difundir información al $17.14 \%$ de los productores. Asimismo, se identificó a los actores que estructuran la red: su importancia es tal que la desvinculación de alguno de ellos debilitaría la red de innovación y conocimiento gestionada; la ausencia de ellos fragmentaria la red en 96.5\% (CEL, técnicos privados y técnicos del gobierno) (Tabla 3).

Tabla 3. Indicadores de redes de los SI analizados.

\begin{tabular}{|c|c|c|c|c|}
\hline $\begin{array}{c}\text { Cadena } \\
\text { agroalimentaria }\end{array}$ & $\begin{array}{l}\text { Centralización } \\
\text { de entrada }(\%)\end{array}$ & $\begin{array}{c}\text { Centralización } \\
\text { de salida }(\%)\end{array}$ & $\begin{array}{c}\text { Difusores de } \\
\text { innovaciones } \\
(\% \text { de } \\
\text { cobertura })\end{array}$ & $\begin{array}{l}\text { Estructuradores } \\
\text { (\% de } \\
\text { fragmentación) }\end{array}$ \\
\hline Bovinos leche & 77.7 & 5.1 & $\begin{array}{c}\text { PC18, PC2, } \\
\text { PC34 } \\
(17.14 \%)\end{array}$ & $\begin{array}{l}\text { Comisión estatal de } \\
\text { la leche, técnicos de } \\
\text { gobierno, técnicos } \\
\text { privados }(96.5 \%)\end{array}$ \\
\hline Ovinos & 6.9 & 1.3 & $\begin{array}{c}\text { POv09, } \\
\text { POv19, POv29 } \\
(5.60 \%)\end{array}$ & $\begin{array}{c}\text { Agencia de } \\
\text { Desarrollo }(90 \%)\end{array}$ \\
\hline
\end{tabular}

PC: Productor de leche / POv: Productor de ovinos. Fuente: Elaboración propia con base en datos de campo 2012-2013. 
El sistema de innovación de bovinos leche se sustenta en la participación de diferentes actores (técnicos que proveen asistencia técnica tanto pública como privada, CEL); entre los cuales resalta la CEL, la cual ha impulsado la adopción de innovaciones. Se trata entonces de un sistema con baja intervención del actor relacionado con la investigación, lo cual es característico de sistemas de innovación en proceso de construcción y de productores pequeños, (Banco Mundial, 2008:190).

Cada sistema es un caso de análisis específico cuyo comportamiento de los actores depende del rol, objetivo e interés específico que tienen en su vinculación con el mercado. La estrategia de intervención para dinamizar y mejorar la generación y difusión de innovaciones, deberá identificar a los actores que funcionan como intermediarios de la innovación. Entendiendo que un intermediario de la innovación es definido como una organización u organismo que actúa como agente o corredor en cualquier aspecto del proceso de innovación entre dos o más partes (Howells, 2006: 720). De acuerdo con Ruiz et al. (2016:131) los intermediarios son quienes operan como puentes entre agentes distantes y la generación de confianza, contribuyendo a un mayor relacionamiento entre los actores.

En la cadena bovinos leche, la CEL, perteneciente a la Secretaría de Desarrollo Agropecuario del estado de Hidalgo, tiene el papel fundamental de fungir como intermediario en la regulación de precios entre los productores de leche y los industriales, basado en la calidad del lacticinio que ofrecen como productores. Estos indicadores sugieren la necesidad de incrementar las interacciones entre los actores de la cadena agroalimentaria a través de la difusión de innovaciones por parte de los generadores de la tecnología e involucrar a las instituciones en este proceso de gestión de la innovación y conocimiento. Los actores detectados como estructuradores o claves dentro de la red son importantes para considerarlos como facilitadores en la transferencia de innovaciones ya que son las fuentes de aprendizaje a las que los productores refieren. El intermediario no solo impacta en el desempeño del sistema de innovación, sino que permite la conformación y orquestación del mismo (Ruiz et al. 2016:130).

Asheim (2005:15) y Banco Mundial (2006:15) definen el Sistema de Innovación como "la infraestructura institucional que apoya a la innovación en la estructura productiva de una región". En este sentido, se puede observar que las fuentes de innovación institucionales del SI bovinos leche son variadas y su incidencia es aún baja, ya que un reducido porcentaje de los productores las referencian como fuente de innovación. Así, Cooke et al. (2003:370) señalan que el sistema de innovación está compuesto por dos subsistemas (subsistema de generación de conocimiento y el

No 19, Vol. 9 (2), 2017. ISSN 2007 - 0705, pp.: 595 - 614 
subsistema de explotación de conocimiento), por tanto, podemos afirmar que la producción de leche en la región se ha desarrollado con base en explotación y uso de los recursos naturales, quedando pendiente una mayor participación del subsistema de generación y explotación del conocimiento (Trippl y Tödtling, 2007:61).

El SI de ovinos se ubica dentro de las trayectorias o patrones de interacción dirigidas en la fase de fundación, debido a que se han identificado a los actores y ha existido apoyo por parte de los agentes gubernamentales mediante un programa de capacitación y asistencia técnica sin llegar a concretar los lazos fuertes entre los diferentes actores. El patrón de interacciones que se presenta en el SI de ovinos está influenciado por la agencia de desarrollo que fortaleció los vínculos a través de la difusión de las innovaciones y el desarrollo de capacidades en los productores, es un sistema que está en proceso de maduración, desarticulado, en donde es necesario seguir fortaleciendo a la agencia como facilitador del proceso de gestión de la innovación. Las interacciones son promovidas por el programa y muy poco se explota el capital de los productores y el conocimiento tácito existente por lo que es necesario el fortalecimiento de la organización de los productores.

Los estudios de casos analizados, han evidenciado diferentes patrones de interacción o trayectorias de innovación asociadas a los actores involucrados, son dos estudios de caso específicos del sector rural en México, los cuales ofrecen elementos para la construcción de sistemas de innovación desde lo local, considerando las potencialidades regionales y necesidades. La evidencia muestra que existen múltiples actores y las conexiones aunque débiles, existen, lo que es verdaderamente importante es conectar a los actores indicados y construir redes en pro de la innovación y algo importante, es necesario considerar las necesidades de los usuarios finales de la innovación.

Los indicadores de redes sociales obtenidos en los casos de estudio sugieren que se trata de un sistema en desarrollo (SI de bovinos leche), con una trayectoria liderada por las oportunidades del mercado, debido a que la CEL actúa como agente facilitador e intermediario entre el productor y la agroindustria y el modelo de desarrollo de proveedores mediante centros de acopio, monitoreo y pago por calidad, con ello se ha logrado la difusión de innovaciones y la mejora, además de contribuir en incrementos en producción y calidad de leche y la especialización lechera de la región. Por otra parte, el SI de ovinos en el Estado de México se asocia con una trayectoria o patrón dirigido, en donde la intervención del gobierno mediante un programa de capacitación y asistencia técnica ha fortalecido la red de productores y el vínculo con diferentes actores del sistema, el riesgo 
de este sistema es que al no consolidarse hasta la etapa de expansión de manera autónoma, al retirarse el Estado puede quedar desarticulado el sistema de innovación, por lo que es necesario una intervención más eficaz de los facilitadores públicos y privados de la innovación.

\section{Interacción de los actores en los SI analizados}

En la perspectiva de los sistemas de innovación, se considera a diversos actores como relevantes para la innovación agrícola, incluidos empresarios agrícolas, investigadores, consultores, formuladores de políticas, proveedores e industrias de transformación, minoristas, clientes (Klerkx and Leeuwis, 2008; 365). De esta forma, es importante conocer a los actores que participan en los SI, pues estos son determinantes en la conformación y funcionamiento del sistema de innovación y permiten identificar a más actores de los que actualmente participan, y que actualmente no están presentes y que pueden representar una diferenciación y ventaja para la innovación. Los resultados permiten identificar coincidencias en algunos actores, que se puede decir son característicos en diversos sistemas de innovación, tales como otros productores, familiares y servicios de asistencia técnica del gobierno (Tabla 4).

Tabla 4. Actores relevantes para la difusión de innovaciones en los SI analizados.

\begin{tabular}{ccc}
\hline Actor/SI & Grado entrada & Grado normalizado (\%) \\
\cline { 1 - 1 } Comisión Estatal de la leche (CEL) & 60 & 81.1 \\
Servicios técnicos de gobierno & 46 & 62.2 \\
Servicios técnicos particulares & 45 & 60.8 \\
Productores & 41 & 55.4 \\
Familiares & 26 & 35.1 \\
& & \\
Productores & Ovinos & 36.2 \\
Familiares & 25 & 21.7 \\
Agencia de desarrollo & 15 & 7.3 \\
\hline
\end{tabular}

Fuente: Elaboración propia con base a datos de campo 2012-2013.

En el caso de bovinos leche, destacan actores estratégicos tales como la CEL quien participa como intermediario entre la agroindustria láctea y los productores y los servicios de asistencia técnica privada quienes fungen de manera estratégica como proveedores de insumos y maquinaría pero también como transferidores de tecnología y fuentes de innovación, esto es más notorio en cadenas como la láctea cuyas características dinámicas y productivas impulsan al productor a adoptar 
nuevas tecnologías e innovar. Del análisis se destaca la falta de actores como las universidades y centros de investigación como fuente de conocimiento, tecnología e innovación; también, es muy baja la incidencia de instituciones de crédito, lo que permite deducir que es necesaria la intervención de actores que dinamicen la generación y transferencia de innovaciones en las cadenas analizadas. En el mismo sentido, los resultados sugieren bajo nivel de interacción lo cual en términos de innovación y aprendizaje es una limitante por lo que se presume es uno de los factores que están limitando el desarrollo del sector rural en México.

\section{Conclusiones}

Los resultados obtenidos sugieren que, en el futuro, los estudios de sistemas de innovación pecuarios aborden, junto con otros elementos, el análisis de la innovación de manera sistémica considerando que en su construcción es necesario evaluar la potencialidad de la región, y su construcción a partir de las redes de colaboración y patrones de interacción existentes entre los productores y actores del sistema.

Las fuentes de aprendizaje e innovación más influyentes en ambos casos son los mismos productores lo que pone de manifiesto el potencial para la difusión de innovaciones entre pares y el aprovechamiento del conocimiento tácito.

Aparecen los intermediarios de la innovación como agentes dinámicos con la capacidad de articular redes locales, vincular a los actores y llevar la innovación hasta el usuario final; estos intermediarios definen las fuentes de innovación y los patrones de interacción en los sistemas de innovación. En el caso de bovinos leche en Hidalgo, la Comisión Estatal de la leche actuó como intermediario y facilitador de la innovación entre la demanda de la industria y la oferta del productor lo cual llevó a adoptar innovaciones para satisfacer la demanda de la industria. En el caso del Sistema de Innovación relacionado con la producción de ovinos en el estado de México, la agencia de desarrollo financiada con recursos del gobierno actuó como el facilitador de la innovación, en donde a partir del diagnóstico participativo se planteó una estrategia de intervención considerando a la innovación como eje central y a partir de las necesidades de los productores se difundieron las innovaciones pertinentes para ellos.

Se identificaron dos mecanismos de innovación; para el caso de la producción de ovinos es una interacción dirigida ya que a partir de la implementación de una política pública y programa se ha mejorado la relación entre los actores; en el caso de bovinos leche se define un mecanismo 
liderado por las oportunidades del mercado en donde a partir de una demanda de la industria y la regulación de la Comisión Estatal de la Leche se ha logrado concertar mejor calidad de la leche y con ello mejor pago por litro del lacticinio.

Para la intervención a través de programas públicos de innovación, transferencia de tecnología y extensión, es necesario el análisis sistémico, haciendo énfasis en la identificación de los actores y facilitadores de la innovación y su potencialidad, los recursos locales para la producción y las necesidades del usuario de la innovación, así como la configuración de las redes y los reservorios de innovación y conocimiento existentes en el territorio. Se concluye que los intermediarios con mayores vínculos definen las fuentes y tipos de innovación, activan los patrones y mecanismos de interacción entre los actores del sistema.

\section{Agradecimientos}

A la Comisión Estatal de la Leche del estado de Hidalgo y los productores de leche que la integran por proporcionar información para el desarrollo de este proyecto. A la Secretaria de Desarrollo Agropecuario del estado de México por el apoyo para la sistematización del modelo de Corredores de Desarrollo Rural implementado en el estado como eje de fortalecimiento de pequeños productores, así como a los productores que proporcionaron información.

\section{Referencias}

Aguilar, A.J.; Rendón, M.R.; Altamirano, C.J.R. 2010. Del extensionismo agrícola a las redes de innovación rural.

Asheim, G. 2005. Contextualizing Regional Innovation Systems in a Globalizing Learning Economy: On Knowledge Bases and Institutional Frameworks. CIRCLE Electronic Working Paper Series n ${ }^{\circ}$ 2005/5.

Banco Mundial. 2008. Incentivar la Innovación Agrícola. Banco Mundial y Mayor Ediciones. Bogotá, Colombia. 204 p.

Borgatti, S.P.; Everett, M.G. y Freeman, L.C. 2002. UCINET 6 for Windows Software for Social Network Analysis. Analytic Technologies, Harvard.1-47 p.

Borgatti, S.P y Halgin D. 2011. On Network Theory. Organization Science. Articles in Advance .doi 10.1287/orsc.1110.0641 
Clark, L. 2006. Creación de capacidades de los agricultores para la creación de redes (Parte II): Strengthening agricultural supply chains in Bolivia using network analysis.Fortalecimiento de las cadenas de suministro agropecuario en Bolivia mediante análisis de redes. KM4D Journal 2(2): 19-32 KM4D Journal 2 (2): 19 - 32

Comisión Estatal de la Lecha del Estado de Hidalgo CEL. 2006. Diagnóstico de producción y rentabilidad de los centros de acopio de leche del sector social en el Estado de Hidalgo. Secretaria de Agricultura y Desarrollo Rural.

Cooke, P.; Roper, S. y Wylie, P. 2003. The Golden Thread of Innovation and Northern Ireland's Evolving Regional Innovation System. Regional Studies. 37 (4):365-379.

Cuevas, R.V., Espejel, G.A., Moctezuma, L.G., Rosales, N. C.A., Tapia, N.A. 2016. Análisis de las redes de financiamiento del sistema nacional de innovación agropecuaria en México. Revista Mexicana de Ciencias Agrícolas. Vol. 7. Número 16. 1297-1309 p.

Espejel, G.A.; Barrera, R.A.; Cuevas, R.V. 2016. Dinámica de innovación y ganancias económicas de la producción de leche en el Valle del Mezquital, Hidalgo. No. 17 Vol. 8 (2). Nova Scientia ISSN 2007-0705. 391-408.

Espejel, G.A.; Cuevas, R.V.; Muñoz, R.M.; Barrera, R.A.; Cervantes, E.F. y Sosa, M.M. 2014. Sistema Regional de Innovación y Desarrollo Rural Territorial; pequeños productores de leche del Valle del Mezquital, Estado de Hidalgo, México. Spanish Journal of Rural Development 5 (2):1-13.

FAO, 2004. The Market for Non-Traditional Agricultural Exports. Roma, Italia. FAO. 163 p.

FAO y Banco Mundial. 2000. Agricultural Knowledge and Information Systems for Rural Development: Strategic Vision and Guiding Principles. Roma, Italia y Washington, DC. FAO y Banco Mundial. 213 p.

Food Agriculture Organization, 2012. Experiencias y enfoques de procesos participativos de innovación en agricultura. El caso de la corporación PBA en Colombia. 55 p. Disponible en http://www.fao.org/docrep/017/i3136s/i3136s.pdf

Fukuda-Parr, S.; Lopes C. y Malik K. 2002. Capacity for Development: New Solutions to Old Problems. New York: Programa de Naciones Unidas para el Desarrollo; Londres: Earthscan. 62 p.

Howells, J., 2006. Intermediation and the role of intermediaries in innovation. Research Policy 35, $715-728$. 
Hall, A. J.; Mytelka L. y Oyeyinka B. 2004. Innovation Systems: Concepts and Implications for Agricultural Research Policy and Practice. Maastricht: United Nations University. 4 p.

Lamprinopoulou, C.; Renwick, A.; Klerkx, L.; Hermans, F.; Roep, D. 2014. Application of an integrated systemic framework for analyzing agricultural innovation systems and informing innovation policies: Comparing the Dutch and Scottish agrifood sectors. Agricultural Systems. 14 p.

Muñoz, R.M.; Aguilar A.J.; Rendón, M.R. y Altamirano, C.J.R. 2010. Análisis de la dinámica de innovación en cadenas agroalimentarias. CIESTAAM-UACh. 40 p.

Muñoz, R.M.; Rendón, M.R.; Aguilar, A.J.; García, M.J.G.; Altamirano, C.R. 2004, Redes de innovación, un acercamiento a su identificación, análisis y gestión para el desarrollo rural. Universidad Autónoma Chapingo, Fundación PRODUCE Michoacán, A.C.

OCDE. 2002. Benchmarking Industry-Science Relationships. 123p. Disponible en http://ep2010.salzburgresearch.at/knowledge_base/oecd_2002.pdf

Raven, R., van den Bosch, S. and Weterings, R. (2010) 'Transitions and strategic niche management: towards a competence kit for practitioners', International Journal of Technology Management, 51: 57-74.

Rendón, M.R.; Aguilar, J.; Muñoz, M. y Altamirano, J.R. 2007. Identificación de actores clave para la gestión de la innovación: el uso de redes sociales. UACh-CIESTAAM-PIAI. 56 p.

Ruiz C. W L., Quintero R. S., Robledo V. J. 2016. Impacto de los Intermediarios en los Sistemas de Innovación. J. Technol. Manag. Innov. 2016. Volume 11, Issue 2: 130-138.

Servicio de Información y Estadística Agroalimentaria y Pesquera 2013. Indicadores estratégicos 15. Sector Agropecuario. Secretaria de Agricultura, Ganadería, Desarrollo Rural, Pesca y Alimentación, SAGARPA. [En línea]. Disponible en: http://www.siap.gob.mx

Smits, R. and Kuhlmann, S. (2004) 'The rise of systemic instruments in innovation policy', International Journal of Foresight and Innovation Policy, 1: 4-32.

Spielman D. J., Ayele G., Davis K., and Negash M. 2008. Rural Innovation Systems and Networks. International Service for National Agricultural Research Division. International Food Policy Research Institute (IFPRI). 1-38 p.

Spielman D. J., Ayele G., Davis K., and Negash M. 2011. Rural innovation systems and networks: findings from a study of Ethiopian smallholders. Agric Hum Values 28:195-212. DOI $10.1007 / \mathrm{s} 10460-010-9273$

No 19, Vol. 9 (2), 2017. ISSN 2007 - 0705, pp.: 595 - 614 
Solleiro-Rebolledo, J. L., Aguilar-Ávila, J., Sánchez-Arredondo, L. G. 2015. Configuración del sistema de innovación del Sector Agroalimentario Mexicano. Revista Mexicana de Agronegocios. enero-junio. v. XIX, (36):1254-1264.

Trippl, M. y Tödtling, F. 2007. Developing Biotechnology Clusters in Non-high Technology Regions-The Case of Austria. Industry and Innovation 14 (1):47-67.

Van Mierlo, B., Leeuwis, C., Smits, R. and Klein-Woolthuis, R. (2010) 'Learning towards system innovation: Evaluating a systemic instrument', Technological Forecasting and Social Change, 77: 318-34.

Vivanco, M. 2005. Muestreo estadístico: Diseño y aplicaciones. Editorial Universitaria. Santiago de Chile. 144 p.

Voss, J.-P., Smith, A. and Grin, J. (2009) 'Designing long-term policy: rethinking transition management', Policy Sciences, 43: 275-302.

Wieczorek, A.J., Hekkert, M.P., 2012. Systemic instruments for systemic innovation problems: a framework for policy makers and innovation scholars. Sci. Public Policy 39, 74-87.

Klerkx, Laurens and Leeuwis Cees. (2008). Balancing multiple interests: Embedding innovation intermediation in the agricultural knowledge infrastructure, Technovation 28 (2008) 364378. 